\title{
Philosophiques
}

\section{L'orientation socialiste dans la philosophie politique de Shelley}

\section{Marie Guertin}

Volume 6, numéro 2, octobre 1979

URI : https://id.erudit.org/iderudit/203118ar

DOI : https://doi.org/10.7202/203118ar

Aller au sommaire du numéro

Éditeur(s)

Société de philosophie du Québec

ISSN

0316-2923 (imprimé)

1492-1391 (numérique)

Découvrir la revue

Citer cet article

Guertin, M. (1979). L'orientation socialiste dans la philosophie politique de

Shelley. Philosophiques, 6(2), 253-271. https://doi.org/10.7202/203118ar d'utilisation que vous pouvez consulter en ligne.

https://apropos.erudit.org/fr/usagers/politique-dutilisation/ 


\title{
L'ORIENTATION SOCIALISTE DANS LA PHILOSOPHIE POLITIQUE DE SHELLEY
}

\author{
par Marie Guertin
}

Shelley, on le sait, est mort noyé en mer, pendant une tempête, le 8 juillet 1822 près de Leghorn en Italie du nord. Ce qu'on sait moins, c'est que la semaine même avant sa mort, il avait entrepris de fonder un journal, qui devait s'appeler The Liberal, avec son ami Leigh Hunt qui venait d'arriver d'Angleterre dans ce dessein, et que précisément il était allé chercher à Pise. Ce journal, auquel il avait fini par convaincre Byron de s'associer, aurait eu pour but de défendre un idéal de liberté sur le double plan de la littérature et de la politique. Cela nous rappelle que Shelley, comme la plupart des grands romantiques anglais (je pense en particulier à Wordsworth, Coleridge et Byron) et d'ailleurs européens, unissait étroitement création poétique et réflexion ou même engagement politique. Mais il y a ceci d'original chez Shelley que, d'une part, il voulait être plus militant, et que surtout, d'autre part, il insiste beaucoup pour que les transformations politiques s'accompagnent d'une réforme sociale en profondeur. Il va si loin en ce sens qu'on peut voir en lui, sans exagération aucune, un véritable précurseur du socialisme'.

1. Le socialisme que l'on trouve chez Shelley, et que le présent article se propose d'étudier, n'est évidemment pas "le socialisme scientifique " de Marx, découverte originale de ce dernier. Car il n'y a chez Shelley ni matérialisme dialectique au sens rigoureux du terme comme conception de l'histoire, ni analyse scientifique du Capital. Mais on ne peut pas dire non plus que le poète anglais se limite à ce quón a appelé après Marx le socialisme utopique et le socialisme réformateur, celui de Fourier par exemple et celui d'Owen. C'est bien une révolution sociale concrète, en rapport avec les conditions de la vie moderne, qu'il réclame, quoiqu'il préfere les voies pacifiques aux voies violentes. En un mot, comme l'a très bien précisé Cameron dans son étude Sbelley: The Golden Years, Shelley se situe à mi-chemin entre le socialisme de son temps et le socialisme marxiste. C'est pourquoi du reste Marx a vu en lui un des principaux précurseurs de ses doctrines. 
Cet élément de la pensée de Shelley, qu'en général le grand public ignore, alors que presque tout le monde connaît au moins quelques-uns de ses grand poèmes, Ode to the West Wind, The Cloud, To a Skylark et Prometheus Unbound, a été étudié en 1974 par un des meilleurs critiques de Shelley, le professeur Kenneth Neil Cameron de l'Université de New York $^{2}$. Mais il avait déjà été perçu avec une clarté parfaite par d'illustres socialistes : Robert Owen, Bernard Shaw, Engels et Marx lui-même, dont la fille prétendait qu'il aurait dit : " La réelle différence entre Byron et Shelley est celle-ci : ceux qui les comprennent et les aiment se réjouissent que Byron soit mort à trente-six ans (sic), parce que, s'il avait vécu plus longtemps, il serait devenu un bourgeois réactionnaire; au contraire, ils regrettent que Shelley soit mort à vingt-neuf ans, parce qu'il était essentiellement un révolutionnaire; et il aurait toujours été à l'avant-garde du socialisme. »Ce à quoi Engels ajoute: «Oui, nous connaissions tous Shelley par cœur $^{3}$.»

Cette même personne, quelques années plus tard, en 1887, donnait une conférence à la Shelley Society où elle dressait le plan d'une étude complète sur le socialisme de Shelley. Nous nous permettons de présenter la traduction de ce plan in extenso, parce qu'on ne saurait mieux faire :

«1. Une note sur Shelley lui-même et sa personnalité, dans sa relation au socialisme. 2. Sur ceux qui, sous ce rapport, ont eu le plus d'influence sur sa pensée. 3. Ses attaques contre la tyrannie et son exaltation de la liberté, dans l'ordre de la pensée. 4. Dans le domaine de l'action. 5. Sa claire perception de la lutte des classes. 6. Son intuition du sens réel que doivent avoir aujourd'hui des mots comme «liberté », « justice », « crime», « travail ", « propriété ». 7. Son attitude pratique, extrêmement pratique, à l'égard des remèdes que requièrent les maux de notre société. 8 . Sa

2. Voir Shelley: The Golden Years, (Harvard University Press), Harvard : 1974.

3. The Shelley Society's Papers, First Series, no. 1, vol. I (Published for the Shelley Society by Reeves \& Turner) London : 1888-1891. Reprint of 1888-1891 (AMS Press) New York: 1975. Cf. "Shelley and Socialism " by Edward Aveling and Eleanor Marx Aveling, Part I, A lecture delivered to the Shelley Society on $14^{\text {th }}$ December 1887 , p. 183 . La traduction des textes de Mme E. Marx Aveling et de Shelley est la mienne. 
compréhension du fait qu'une reconstruction de la société est inévitable, en même temps qu'imminente. 9. Ses descriptions du futur, utopies qui ne sont pas des utopies. 10. Enfin, une allusion à ses principaux travaux dans lesquels ses idées socialistes ont trouvé leur expression ${ }^{4}$. $\gg$ Bien entendu, il n'est pas question, dans cet article, d'aborder tous les sujets qui viennent d'être énumérés. Nous nous contenterons d'aborder, d'ailleurs brièvement, les trois points suivants : (1) la lutte des classes ; (2) les principes de justice et d'égalité sur lesquels doit être basée la réforme de la société ; (3) la philosophie de la révolution. Cela suffira pour montrer, nous l'espérons, combien la philosophie sociale de Shelley, quoiqu'elle n'ait été exprimée que dans des écrits d'occasion par un homme mort à 29 ans, contient déjà une véritable esquisse d'un socialisme par certains côtés avant-coureur du socialisme de Marx, mais par d'autres côtés très différents de ce dernier ${ }^{5}$.

\section{LA DÉCOUVERTE DE LA LUTTE DES CLASSES EN IRLANDE ET EN ANGLETERRE D'ABORD, PUIS DANS L'HISTOIRE DE L'HUMANITÉ EN GÉNÉRAL}

Comme on le sait, Shelley, après avoir écrit son Essay on Atheism au mois de mars 1811, est expulsé d'Oxford. Il refuse, dès l'automne de cette même année, de remplir les fonctions de membre du parlement pour les Left-Whigs, position que lui offrait le duc de Norfolk, ami fidèle de la famille des Shelley. Mais dès le mois de décembre le jeune homme pense aller en Irlande, pays qui l'intéresse particulièrement à cause des graves problèmes qui s'y présentent ${ }^{6}$. Il est bon de se rappeler qu'en 1801 William Pitt, alors premier ministre, avait accordé à l'Irlande le fameux Acte d'Union en pensant régler la situation des Irlandais. Mais rien n'avait été réglé et la situation allait en empirant. Si donc Shelley s'achemine vers l'Irlande en février 1812 et se décide à y rester six semaines,

4. Ibid., p. 183.

5. Ici encore voir Cameron, Shelley : The Golden Years, p. 131: "He stands, in fact, about halfway between Godwin and Marx ", et, pour plus de précision, p. 136 à 137.

6. Pour les références à la vie de Shelley et à ses essais philosophiques politiques, voir les deux ouvrages suivants : The Letters of Percy Bysshe Shelley, in two volumes, ed. by F.L. Jones, (Oxford at the Clarendon Press) Oxford: 1964. Shelley's Prose or The Trumpet of a Prophecy, ed. by D. Clarke, (Alburquerque Press) New Mexico : 1954. 
c'est pour essayer de trouver un remède à la crise en parlant aux gens du peuple. Dans ce but il compose deux essais : Address to the Irish People, Proposals for an Association of Philantbropists, ainsi qu'une charte Declaration of Rights, écrite à Dublin mais qu'il ne pourra faire parvenir aux Irlandais ${ }^{7}$. Il désirait tellement s'adresser de façon directe au peuple qu'il dit dans une lettre écrite à une amie, Élizabeth Hitchener : " Mon adresse sera imprimée sur un papier de qualité inférieure et en lettres très fines pour raccourcir le nombre de pages et ainsi alléger le prix de vente pour les Irlandais ${ }^{8}$. "En plus de vouloir vendre ses articles à bas prix, il s'applique à adapter son langage à celui du peuple. C'est ce qu'il explique dans une autre lettre adressée cette fois à William Godwin, le célèbre philosophe socialiste, auteur du Enquiry Concerning Political Justice : "J'ai volontairement vulgarisé le langage dans ce pamphlet afin de réduire les remarques qu'il contient au goût et à l'entendement des paysans Irlandais qui ont été trop longtemps brutalisés par le vice et l'ignorance ${ }^{9}$. " Mais ce qu'il y a encore de plus touchant, c'est que le poète veuille faire quelque chose de pratique pour les Irlandais, car il sent la précarité de leur état : «C'est l'intention de l'auteur d'éveiller dans les esprits des pauvres Irlandais la connaissance réelle de leur situation, leur indiquant les maux de cet état et leur suggérant des moyens rationnels pour y remédier ${ }^{10}$." Mais Shelley ne veut pas être seul dans sa campagne à Dublin et il invite à plusieurs reprises son amie, Élizabeth Hitchener, à les rejoindre, lui et son épouse, pour travailler à cette grande cause. Tout en se préparant pour le grand jour où il rencontrera les gens du peuple, il met en pratique son idéal de réforme sociale en soulageant quelques familles mourant de faim dans le quartier où il vit temporairement. Il réussit à distribuer autour de lui 400 à 600 copies de ses articles et il en pose sur les murs de la ville. Le 28 février, Shelley se rend au théâtre de Fishamble Street pour assister à la réunion des

7. Sous les pressions de William Godwin, d'une part, et à cause du mécontentement de certains Irlandais, Shelley dût quitter la ville avant de distribuet cette charte qu'il comptait voir afficher dans toutes les maisons des Irlandais.

8. The Letters of Periy Byssbe Shelley, I, no 161 : Jan. 16, 1812, p. 235.

9. Ibid., no 170 , p. 258.

10. Shelley's Prose or The Trumpet of a Prophecy, "Address to the Irish People ", p. 39 
Irlandais catholiques et, à cette occasion, il prend la parole pendant une heure.

Ce qu'il essaie de montrer dans les premiers moments de son discours, c'est tout d'abord sa profonde sympathie pour ses auditeurs, car ils sont ses frères qui souffrent. Il voudrait apaiser les animosités qui opposent Irlandais et Anglais, en rappelant que le conflit n'est pas dû̀ à une mésentente congénitale entre les deux ethnies, car l'Angleterre « poursuivrait la même politique avec n'importe quel pays du monde, jusqu'au jour où les hommes qui subissent son hégémonie se décideront à demander des changements et ne se laisseront plus mener ainsi ${ }^{11} \gg$. Shelley souligne ensuite qu'il n'ignore pas que les autorités de Dublin, en lisant le titre de sa conférence, auront l'espoir d'y trouver une incitation à la violence, qui leur donnerait occasion de poursuites devant les tribunaux. "Ces hommes sont méchants, continue-t-il, et tout ce qu'ils méritent, c'est le mépris des bons, qui doivent éviter de nuire à leur cause par un excès d'indignation ${ }^{12}$." Ainsi l'orateur assure les paysans que son adresse ne contiendra rien qui puisse affaiblir la cause de la liberté, car elle ne fera que prêcher la modération et la tolérance.

Les deux plus grandes causes de l'injustice qui règne en Irlande, telles que Shelley les dénonce, sont les suivantes : la non-reconnaissance par le gouvernement anglais de la religion catholique que les Irlandais pratiquent ; l'Acte d'Union entre l'Irlande et l'Angleterre. Nous nous bornerons à évoquer très sommairement ces deux points, quoique les considérations du jeune Shelley reprennent aujourd'hui une extraordinaire actualité, parce que ce que je voudrais surtout souligner c'est la façon dont il rattache à ces problèmes politico-religieux des problèmes sociaux. Au point de vue religieux, Shelley dénonce essentiellement les guerres de religion qu'il juge absurdes et en contradiction avec le christianisme: "S'ils avaient vu un pouce plus loin que leur nez, dit-il, en parlant des sectaires acharnés à se combattre, ils auraient compris que battre et tuer des hommes était le pire moyen pour s'attirer la faveur d'un 
Être (Dieu) à qui on ne plaît, c'est l'aveu de tous, que par des actions d'amour et de charité ${ }^{13}$. "Il faudrait que les catholiques acceptent les protestants et les protestants les catholiques en rivalisant seulement dans la pratique de la justice, car ce qui les sépare, affirme le jeune poète, ne compte guère. Et c'est en vue d'instaurer la tolérance religieuse et de la perpétuer dans les générations futures que Shelley voudrait installer en Irlande son Association de Philanthropes.

Avec la question de l'Acte d'Union nous touchons plus directement aux problèmes sociaux qui aggravent le problème politique. Car la conséquence la plus palpable de l'Acte d'Union est d'avoir garanti à l'aristocratie foncière d'origine anglaise son implantation en Irlande, qui lui permet d'exploiter ce pays déjà pauvre et de l'appauvrir davantage. Shelley dénonce cette situation dans une formule lapidaire: "L'aristocratie installée en Irlande suce les veines de ses habitants et va boire leur sang en Angleterre ${ }^{14}$. "C'est pourquoi le paysan irlandais est devenu si malheureux tandis que quantité de mendiants traînent dans Dublin, que la famine se répand et que le crime augmente. Voilà pourquoi les Irlandais sont désespérés et révoltés. Mais les autorités, du roi aux ministres, mentent lorsqu'elles prétendent que l'Irlande est incapable de se gouverner elle-même sans sombrer dans la révolution et dans l'anarchie. C'est le contraire qui est vrai. L'indépendance serait bénéfique aux Irlandais. Si le gouvernement anglais la leur refuse, c'est parce qu'il est au service de ceux qui veulent exploiter ce peuple pour leur propre profit. C'est ainsi que Shelley montre que les rapports politiques ne sont, en fait, dans le cas de l'Irlande que l'expression superficielle et déformée de rapports de classes plus profonds et plus importants. Ces mêmes rapports de classes, ou du moins des rapports analogues, Shelley les retrouve autour de lui en Angleterre.

Cela n'a rien d'étonnant, puisque le gouvernement de l'Angleterre agit de la même façon avec les siens qu'avec ses voisins d'Irlande. Cette lutte de classes est vivement décrite par le poète dans plusieurs petits essais tels que : And Statesmen

13. lbid., p. 42.

14. Ibid., "An Association of Philanthropists ", p. 62 
Boast of Wealth (1818); A Proposal for Putting Reform to the Vote Throughout the Kingdom (1817); An Address to the People on the Death of Princess Charlotte (1817). Mais cette bataille entre les riches et les pauvres, Shelley la peint et il définit mieux ses causes dans l'essai A Philosophical View of Reform, écrit en 1818, et c'est à cet essai que nous allons nous référer le plus souvent dans notre analyse, car c'est le meilleur écrit politique du poète-philosophe.

Il faut se rappeler qu'une période de 7 à 9 ans sépare l'Irlande de 1812 de l'Angleterre de 1817-18 et que la révolution industrielle s'est beaucoup implantée dans le Yorshire et le Lancashire. Or cette révolution industrielle a le plus souvent engendré, contrairement à ce que prétendaient les économistes libéraux, une aggravation de la misère. On a fait des promesses aux prolétaires en leur faisant miroiter un gain plus lucratif s'ils venaient travailler dans les manufactures. Mais en fait ce n'est pas le beau rêve tel que promis qui s'est réalisé. "Les manufacturiers ont demandé aux hommes, aux femmes et aux enfants de travailler non plus 8 heures par jour comme autrefois mais 10,12 et jusqu’à 14 heures par jour, et sur ces 12 ou 14 heures à peine 7 se trouvent payées effectivement $^{15}$. $\gg$ Le reste passe dans la poche du capitaliste. C'est déjà l'anticipation de ce que Marx appellera la plus-value du capital fondée en grande partie sur du travail non payé. Et Shelley insiste aussi sur la baisse qu'a entraînée la production manufacturière dans la valeur du travail : en gros, il faut maintenant travailler 20 heures pour acheter ce qu'on se procurait autrefois avec 10 heures de travail, et c'est en quelque sorte pour parvenir à accumuler dans une famille 20 heures de travail par jour là où il en fallait 10 , que les enfants et les personnes âgées sont eux aussi obligés de travailler jusqu'à l'épuisement de leurs forces. Ceux qui deviennent trop fatigués par le travail sont forcés de travailler ou de mourir de faim. Et lorsque ceux-ci ne peuvent se procurer du pain parce qu'ils ne peuvent plus travailler, ils essaient de le voler ${ }^{16}$. Si bien que Shelley nous dit dans son Philosopbical View of Reform: «The majority of the people of England are destitute and

15. Ibid., "A Philosophical View of Reform ", p. 244, 246.

16. Ibid., p. 246. 
miserable, ill-clothed, ill-fed, ill-educated ${ }^{17}$. " De là vient qu'ils sont immoraux, misérables et désespérés. Et pourtant ils forment la classe la plus nombreuse de la société et celle qui par son labeur nourrit et habille toutes les autres. Car, et ici nous citons encore : " il n'y a pas d'autre réelle richesse que le travail de l'homme ${ }^{18}$ ". Si donc la classe qui travaille vit dans la misère, c'est que la société s'est installée dans l'injustice et la contradiction. Ces quelques extraits que nous venons de résumer attestent avec évidence que Shelley est déjà un écrivain socialiste par la netteté avec laquelle il voit, par la profondeur avec laquelle il ressent la misère des classes laborieuses dans la société anglaise contemporaine . . . Si bien qu'en le lisant on pense inévitablement à ce que Engels devait écrire quelques 25 ans plus tard dans le premier grand ouvrage de la pensée marxiste : La Situation des classes laborieuses en Angleterre ${ }^{19}$. Je cite Engels, mais c'est tout aussi bien Robert Owen, Louis Blanc, Proudhon etc. ${ }^{20}$, bref tous les penseurs socialistes que Shelley devance en dénonçant, comme il l'a fait, la misère de la classe paysanne et de la classe ouvrière. Mais le poète-philosophe devance également le socialisme sur un autre point capital : car il a aperçu clairement l'avènement de la bourgeoisie comme classe dirigeante. Il appelle cette bourgeoisie la deuxième aristocratie pour bien souligner, comme Marx le fera plus tard, qu'elle supplante l'ancienne aristocratie de la noblesse héréditaire, comme l'ère industrielle avec laquelle cette nouvelle aristocratie a partie liée supplante la civilisation de la charrue et du moulin à bras. Laissons la parole à Shelley que nous nous bornerons à traduire en citant un court passage de son Philosopbical View of Reform auquel on pourrait en ajouter bien d'autres : "La conséquence de ces changements a été l'établissement d'une nouvelle aristocratie, fondée sur la fraude comme l'ancienne l'a été sur la force. Et permettez-moi d'employer le mot ARISTOCRATIE dans cette acception

\footnotetext{
17. Ibid., p. 247

18. Ibid., "And Statesmen Boast of Wealth", p. 113

19. La Situation des lasses laborieuses en Angleterre, par F. Engels, 2 tomes, traduit par Barache, A-M. Desrousseaux, et D. et P.J. Berthaud (Alfred Costes) Paris : 1933.

20. Voir Cameron, Shelley : The Golden Years, ch. 3: "Political Philosophy ", p. 115-149; et Les Précurseurs Français du Socialisme. De Condorcet à Proudbon, Textes réunis et présentés par M. Leroy, (Édirions du temps présent) Paris : 1948.
} 
commune, à savoir une classe de personnes qui possèdent un droit à s'emparer des fruits du travail des autres sans s'obliger en retour à servir la communauté par quelque travail que ce soit $^{21}$. " Et Shelley souligne ensuite que la nouvelle aristocratie, plus rapace et plus dure que l'ancienne, lui est aussi inférieure en fait de courage, de générosité et d'élégance. Ce qui fait qu'elle ne concourt ni à servir ni à orner l'ensemble de la société : elle s'applique surtout à l'exploiter. La principale vertu de cette aristocratie de parvenus est en effet de savoir accumuler des biens qu'ils n'ont pas mérités. Car pour Shelley il faut distinguer et opposer deux formes de propriété. L'une est acquise par " le labeur, l'industrie, l'économie, l'exercice du talent personnel ou même du génie, bref, par toute espèce de moyens honorables et honnêtement utilisés ${ }^{22} »$. La propriété obtenue par ces moyens est tout à fait justifiable.

Mais il y a d'autres façons d'obtenir des biens et de parvenir à la propriété, qui, elles, «représentent autant de voies frauduleuses et violentes, ne reculant ni devant l'imposture ni devant l'usurpation, parce que leurs auteurs ne s'embarrassent pas des droits d'autrui mais cherchent seulement leur profit maximum sans aucune autre considération $^{23}$ ». Et sur ce point Shelley se rapproche beaucoup des idées exprimées par Engels, dans le chapitre sur « La bourgeoisie anglaise " de l'ouvrage que j'ai déjà eu l'occasion d'évoquer: La situation des classes laborieuses en Angleterre ${ }^{24}$. Car la classe qui a fait de l'accumulation du profit et de la propriété la fin suprême de l'activité humaine, et qui cherche à agrandir sa fortune sans vergogne, cette classe vit de l'exploitation de ceux qu'elle fait travailler dans ses entreprises; il en résulte, avec l'injustice faite aux travailleurs, une misère sociale d'ensemble qui mine la société jusque dans ses fondements. Car l'appétit insatiable de profit, qui domine la bourgeoisie et s'est aussi répandu dans l'ancienne aristocratie, est la cause de la misère noire dans laquelle une majorité du peuple se débat. Et la misère qu'engendrent ces appétits effrénés n'est pas seulement

\footnotetext{
21. Shelley's Pruse or The Trumpet of a Propbecy, "A Philosophical View of Reform », p. 245

22. Ibid., p. 250 .

23. Ibid., p. 250 .

24. Engels, F., La Situation des classes laborietses en Angleterre, p. 243-247.
} 
matérielle : elle est intellectuelle et morale aussi, puisque la classe des travailleurs est maintenue dans un état proche de l'état sauvage. Autant d'idées qu'Engels analysera profondément et surtout illustrera par des milliers d'exemples, mais qu'on trouve déjà énoncées par Shelley avec beaucoup de force et de clarté.

En outre Shelley montre avec force comment le système politique et le système financier favorisent cette inégalité sociale. Des deux Chambres qui constituent le Parlement anglais et sont censées faire du gouvernement anglais un régime démocratique, l'une, la Chambre des Lords, ne représente en fait que l'aristocratie, puisque ses membres s'y trouvent par droit héréditaire ou par la faveur du roi ; l'autre, la Chambre des Communes, représente en droit le peuple; mais le système d'élection qui prévaut dans le pays fait que seule la classe bourgeoise y est représentée. En conséquence les lois qui y sont votées sont adoptées dans l'intérêt de cette classe bien plus que dans l'intérêt du peuple. La pratique aggrave ce que les institutions permettent, et Shelley cite comme un exemple, sur lequel il insiste beaucoup, le Massacre de Peterloo en août 1818 à Manchester, où les ouvriers qui manifestaient de façon non violente ont été dispersés brutalement par l'armée. Onze personnes ont été tuées, hommes, femmes et enfants ${ }^{25}$. Et quand la répression n'est pas aussi ouvertement brutale, elle n'est pas moins violente et injuste pour être plus dissimulée. Shelley l'explique longuement dans l'essai qu'il a écrit sur la mort de la princesse Charlotte, événement à l'occasion duquel deux ouvriers victimes d'un agent provocateur avaient été envoyés à l'échafaud. Une deuxième cause d'inégalités est la politique de guerre qu'a poursuivie le gouvernement anglais avec l'appui des riches, grâce au système financier qui est lié au système politique. Pour financer la guerre qu'il a soutenue en Amérique d'abord, puis contre la France pendant plus de 20 années, le gouvernement anglais a dû consentir des emprunts considérables. Cela fait peser sur la nation une dette énorme qui, en outre, s'accrôt chaque année des gros intérêts qu'il faut payer aux

25. Shelley a écrit un poème à ce sujet : The Mask of Anarby, en 1819 . 
financiers. Mais qui rembourse la dette et qui paye ces intérêts? Pour l'essentiel, ce sont les citoyens soumis à la taxation et en particulier les travailleurs pauvres qui fournissent la grosse part de l'impôt, alors que les riches, qui sont des créanciers plus encore que des contribuables, reçoivent dans leur poche les intérêts de la dette. Ainsi ils s'enrichissent de la guerre pendant que les pauvres s'appauvrissent. Ajoutons, car Shelley l'ajoute, que la substitution de la monnaie de papier à la monnaie d'or a permis l'aggravation de tous ces maux, en particulier parce qu'elle favorise inflation et dévaluation. C'est ainsi que le système et la pratique politiques qui prévalent en Angleterre contribuent à aggraver la misère du peuple produite par la situation sociale.

Cette lutte de classes qu'il a si bien aperçue autour de lui, Shelley la retrouve à travers l'histoire ${ }^{26}$. Dans le grand événement tout d'abord qui avait marqué son enfance et sur lequel il n’a jamais cessé de méditer : à savoir la Révolution française dont il a partagé les idéaux et dont il déplore l'échec. L'avènement de Napoléon, en particulier, lui a semblé chose funeste, non seulement parce qu'il a signifié la guerre en permanence et l'abolition de la république au profit de la dictature, mais encore parce qu'il a consacré la victoire des riches sur les pauvres. Or la Révolution française n'est en définitive que l'exemple le plus proche et le plus grandiose de ce qui s'est sans cesse produit depuis que les hommes vivent en société. Et dans les premières pages de son Philosophical View of Reform, Shelley dresse un tableau saisissant de la lutte entre pauvres et riches, oppresseurs et opprimés, depuis la dissolution de l'Empire romain jusqu'à la Révolution américaine, en passant par l'évocation de l'État féodal au Moyen Âge, des guerres entre les cités italiennes et des combats qu'ont dû affronter les républiques de Suisse et de Hollande, ou les réformateurs en Angleterre. Nous ne nous attarderons pas sur ce tableau composé d'ailleurs de façon très rapide, qui demeure pour cette raison passablement abstrait. Il nous suffira de

26. Le poète était un passionné de lectures historiques : le journal de son épouse où sont inscrites souvent ses lectures selon les années nous démontre bien cet intérêt. Voir aussi l'effet de la Révolution française sur Shelley dans le livre : Shelley and the Revolutionary Idea, de G. McNiece, (Harvard university Press) Harvard : 1969. 
souligner ce qui s'en dégage avec évidence : pour Shelley, la lutte de classes entre pauvres et riches ainsi que la recherche d'une libération par les opprimés sont, en tous pays et en tous temps, des éléments essentiels de l'histoire. Or cette idée que Shelley expose abstraitement dans ce traité, on la retrouve sous forme symbolique dans nombre de ses poèmes. Ainsi dans le très long poème The Revolt of Islam, longue méditation dramatique et métaphorique sur la Révolution française et la révolution en général, et dans Ode to Liberty, Ode to Naples, Hellas, que nous évoquerons en quelques mots. Et il faudrait en citer bien d'autres ; par exemple : Queen Mab, To the People of England et surtout The Mask of Anarchy. Ce dernier poème, inspiré d'ailleurs par le "Peterloo Massacre ", résume symboliquement tous les thèmes que nous venons de mentionner. Car le masque, ce sont les diverses autorités, églises, gouvernement, justice, armée, que Shelley fait défiler dans une étrange procession. Ces autorités, qui sont mises en place pour combattre l'injustice, du moins à ce qu'on dit, l'aggravent au contraire et sont les vrais facteurs d'anarchie et de désordre, sous le manteau de dignité qui les cache. Les autorités gouvernantes accusent les classes populaires de créer l'anarchie; mais ces dernières en sont les victimes, et les vrais accusés devraient être les riches, l'aristocratie, la bourgeoisie, la monarchie, en un mot les spoliateurs et les oppresseurs de tout genre.

\section{LES PRINCIPES DE JUSTICE ET D'ÉGALITÉ SUR LESQUELS DOIT ÊTRE BASÉE LA RÉFORME DE LA SOCIÉTÉ}

Shelley, nous venons de le voir, a mené une critique acharnée contre les injustices sociales et politiques de son temps et de son pays. Mais il a su aussi apporter des vues positives sur la réforme qu'il désirait et proposer une série de mesures concrètes pour améliorer le sort du peuple en mettant plus de justice dans la société. Les premiers principes sur lesquels doit se fonder la réforme, dit le poète, sont en effet ceux de la justice et de l'égalité27. Ces idées sont principale-

27. Il est impossible de rappeler dans ce court article les traits de la société idéale telle que Shelley l'a conçue. Mais il est certain qu'il a constamment cherché à s'en former la vision la plus claire possible à partir des principes de justice, d'égalité et de liberté. On peut 
ment développées dans deux nouveaux essais : l'un sur l'éthique, A Treatise on Morals, et l'autre sur la religion, Essay on Christianity. Shelley enseigne dans son traité de morale "que l'idée de justice est, comme la bienveillance, une loi élémentaire de la nature humaine ${ }^{28} »$. Ou, plus exactement, cette idée est la règle qui nous fait diriger nos actions conformément à un sentiment de bienveillance envers les autres. Et ce qu'elle prescrit de la façon la plus immédiate, c'est un partage égal des biens entre tous les hommes. Pour illustrer ce qu'il veut dire par une image simple et forte, Shelley compare l'humanité à un groupe de naufragés qui se sont réfugiés sur une île déserte. "Ils distribuent entre eux, et en parts égales, tout ce qu'il leur reste de subsistance. Si quelques-uns d'entre eux conspirent pour priver les autres de ce qui leur revient, leur conduite sera qualifiée d'injuste ${ }^{29}$. "Ce que Shelley veut montrer par cet exemple, c'est que l'égalité est une idée naturelle chez l'homme et dans la nature : "Nature made men all alike. " Il répète des formules semblables à maintes reprises dans sa correspondance. Donc, pour lui, si les hommes partent de ce principe pour réfléchir sur leur système social et politique, ils verront combien le fait d'avoir différentes classes sociales ne peut être supporté plus longtemps. Cette division entre les riches et les pauvres est mauvaise et dénaturée, car elle permet la domination d'une classe au détriment de l'autre, comme Shelley l'avait souligné dans son Adresse aux Irlandais : " $\mathrm{Na}$ ture never intended that there should be such a thing as a poor man or a rich one ${ }^{30}$." Non seulement cette division est injuste et contraire à la nature, mais elle est source d'aliénation aussi bien pour la classe riche que pour la classe pauvre, toutes les deux se trouvent placées dans une situation non naturelle, qui empêche le bonheur des uns et des autres. Ici encore, il faut citer le jeune réformateur: "Dans notre société, dit-il, le pauvre nait pour servir le riche, bien que tous les deux arrivent

s'en rendre compte aussi bien à travers ses lettres et ses écrits philosophiques qu'à travers sa poésie et son action militante. La poursuite d'un idéal de justice est une de ses inspirations majeures, comme j'ai essayé de le montrer dans ma thèse : Shelley's Poetic Inspiration and its Tu'n Sources : the Ideals of Justice and Beauty, Universite d'Ottawa 1977, $164 \mathrm{p}$.

28. Shelley's Prose or The Trumpet of A Prophecy, "A Treatise on Morals", p. 190.

29. Ibid., p. 190.

30. Ibid., "Address to the Irish People ", p. 50. 
dans le monde également dépourvus et également nus. Mais le pauvre ne rend en réalité aucun service au riche en lui obéissant, et le riche ne fait aucun bien au pauvre en le dominant ${ }^{31}$."

Pour remédier par l'égalité à cette aliénation générale, Shelley propose essentiellement deux types de réforme. Premièrement, une réforme politique dans le sens d'une démocratie véritable et complète. Il faut en particulier, dit-il, que la représentation du peuple devienne majoritaire à la Chambre des Communes, comme elle l'est dans le pays. Et Shelley souhaiterait vivement que l'Angleterre cesse d'être une monarchie pour devenir une république, gouvernée par une seule Assemblée, celle du peuple. En d'autres mots, il voudrait installer un régime complètement fondé sur la souveraineté populaire qui ne doit avoir d'autre limite selon lui que le respect des droits de chaque personne et de chaque groupe. Notons cependant que Shelley sait modérer sur ce point son idéal réformateur. Il voit bien que pour le moment le peuple anglais ne désire pas supprimer la monarchie et installer une république. En outre, il craint qu'un changement brusque nuise à la réforme et il préfere une démarche plus progressive. C'est pourquoi il se contenterait d'obtenir un aménagement des institutions monarchiques et parlementaires telles qu'elles existent.

La deuxième réforme fondamentale que Shelley propose touche à la répartition des richesses et du travail. En ce qui concerne le premier point, Shelley modère ou adapte aux réalités son idéal égalitaire comme il a su y adapter son idéal démocratique. Il ne cherche pas à atteindre une complète égalité des biens qui ne serait possible qu'avec l'arrivée de la perfection sur la terre : "Equality in possessions must be the last result of the utmost refinements of civilization ${ }^{32}$. » "C'est l'état de la société vers lequel il est de notre devoir de tendre, tout en sachant qu'on est fort loin de pouvoir $y$ parvenir. Mais cette modération et ce réalisme ne doivent pas cacher l'urgence des réformes qu'il faut entreprendre avec

31. 1bid., p. 50.

32. Ibid., "A Philosophical View of Reform », p. 253. 
vigueur sans plus $\operatorname{tarder}^{33}$. » Et c'est ainsi que Shelley demande avec force que les riches paient par l'impôt sur leurs revenus la dette nationale que leur politique de guerre a entrainé le pays à contracter. De même il faudrait opérer tout de suite un nouveau partage des terres et une redistribution des propriétés, en modifiant en particulier les lois sur l'héritage : de la sorte on pourrait donner aux cultivateurs pauvres ces immenses terres que les riches propriétaires ont en trop.

Sur le travail, l'idée essentielle de Shelley est celle qu'il résume dans cette formule frappante : le travail est la propriété la plus sacrée de l'homme et la source de toute autre propriété : «a property of the most sacred and indisputable right and the foundation of all other property ${ }^{34}$." C'est pourquoi il est aussi bon que juste que tous les hommes soient contraints de travailler. De même chaque homme doit pouvoir exercer son droit de travailler. En un mot, Shelley a sur le droit au travail les idées que Louis Blanc devait essayer d'appliquer en France pendant la révolution de $1848^{35}$. Mais le poète-philosophe entretient sur ce point une autre idée chère aux socialistes : c'est que l'homme étant indivisiblement âme et corps, muscles et pensée, il devrait y avoir équilibre chez lui entre le travail physique et l'activité intellectuelle, que Shelley, conformément à la tradition aristocratique des anciens, appelle le loisir : "Labor is required for physical, and leisure for moral improvement ${ }^{36}$. " "Eviter au riche le travail physique, c'est le condamner à une débilité qui aliène sa nature d'homme, comme l'aliènent la jouissance de richesses excessives et la domination sur les autres. Mais priver le pauvre de loisir est encore plus grave, car c'est lui ôter toute possibilité d'amélioration intellectuelle et morale ${ }^{37}$."

\section{LA PHILOSOPHIE DE LA RÉVOLUTION}

On pourrait sans doute apporter sur tous les points que nous venons d'envisager bon nombre de précisions supplé-

\footnotetext{
33. Ibid, , p. 253.

34. Ibid., p. 251.

35. Les Précurseurs Français du Socialisłe, p. $325-348$

36. Shelley's Prose or The Trumpet of a Propbecy, "And Statesmen Boast of Wealth », p. 114.

37. Ibid., p. 114.
} 
mentaires. Mais nous passons sans plus tarder au dernier point que nous voulons examiner, pour comparer au socialisme la philosophie politique de Shelley: à savoir son idée de la révolution. Cette idée ne joue pas un moindre rôle dans sa pensée que celle de la lutte des classes et du principe de l'égalité. Mais nous nous bornerons à en considérer brièvement deux aspects : (1) comment l'idée de la révolution est liée à un intérêt très vif pour les révolutions en cours; (2) comment Shelley voudrait voir la révolution adopter la non-violence, même s'il semble reconnaitre dans telle ou telle de ses lettres que le recours à la violence est parfois difficile à éviter ${ }^{38}$.

En 1817, l'exil du poète en Italie n'a pas changé son intérêt pour la politique et la société, ni son idéal de justice sociale. Une lettre du 6 novembre 1819 à Hunt, entre autres, indique clairement que sept ans après son intervention en Irlande il continue de s'intéresser à la révolution et qu'il garde son cœur aux révolutionnaires : "Nous vivons, écrit-il à son ami, des temps terribles... Et nous ne pouvons hésiter sur le parti à embrasser : quelques révolutions qui puissent arriver, sous quelque nom que se cache l'oppression, qui reste toujours l'oppression, notre parti sera celui de la liberté et des opprimés ${ }^{39}$. "Comme si les événements voulaient venir au devant de ses sentiments, trois révolutions allaient éclater successivement en Europe ${ }^{40}$. La première, le $1^{\text {er }}$ janvier 1820 , en Espagne, qui s'acheva en juillet ; la deuxième, qui débuta à l'automne de 1820, à Naples, et se termina en mai 1821 ; et la dernière qui se produisit à la même date en Grèce, et continua jusqu'en 1828 , c'est-à-dire six ans après la mort de Shelley. Comme on le sait, deux de ses amis, Trelawny et l'illustre Byron, iront se battre, inspirés par son enseignement, sur la terre hellénique. Quant à Shelley lui-même, il était mort trop tôt pour pouvoir participer à leur expédition, et auparavant sa trop faible santé l'avait empêché de se joindre, comme il l'aurait voulu, aux révolutionnaires espagnols et napolitains. Mais, pendant toute cette période, on le voit lire avidement les

\footnotetext{
38. The Letters of Percy Bysshe Shelley, II, no 585, p. 234.

39. Ibid., no 258 , p. 148.

40. Pour une connaissance brève mais claire des révolutions en Italie, en Espagne er en Grece, voit Cameron, Sbelley: The Golden Years, ch. 10: "The Lightning of the Nations ", p. 363-393.
} 
journaux qui lui permettent de suivre ces événements. De surcroît, il cherche à obtenir de gens plus proches des lieux où ces événements se déroulent toutes espèces de témoignages directs, et sa correspondance de l'époque est toute remplie de ses espoirs, de ses craintes, de ses doutes. Il est si enthousiasmé et si accaparé que son entousiasme et ses soucis se répercutent sur son épouse, Mary Godwin Shelley, qui à son tour écrit des lettres enflammées sur l'avènement prochain de la réforme. Quant à lui, faute de devenir une espèce de " chevalier moderne " à la tête des révolutionnaires, il leur dédie trois grands et beaux poèmes : Ode to Liberty, Ode to Naples, Hellas. Nous ne pouvons nous attarder sur les idées contenues dans ces poèmes, ni en analyser la beauté. Nous nous bornerons, pour illustrer la passion révolutionnaire de Shelley, à indiquer leur fil directeur. Dans Ode to Liberty ${ }^{41}$, le poète montre essentiellement trois idées : (1) Il exalte le peuple espagnol qui se bat pour la cause de la liberté : "A glorious people vibrated again/The lightning of the nations/From heart to heart, from tower to tower, O'er Spain/Scattering contagious fire into the Sky, Gleamed » (I, 1-5). (2) Il retrace, à travers l'histoire primitive, ancienne et européenne, les bonds et les reculs de la liberté chez les différents peuples et nations. (3) Il appelle le retour des esprits philosophiques et poétiques et prie la liberté de s'installer non seulement en Espagne, mais chez tous les hommes. On retrouve des idées analogues dans Ode to Naples et dans le poème dramatique Hellas. Dans Ode to Naples ${ }^{42}$, Shelley glorifie la beauté naturelle de Naples, mais il pleure la disparition en l'homme de la beauté originale, c'est-à-dire morale. Il encourage le peuple à reconquérir de nouveau le règne de l'espoir, de la vérité et de la justice, et implore l'Esprit d'Amour et de Beauté de pénétrer cette ville et tous les hommes à nouveau. Quant à $\mathrm{Hellas}^{43}$, qui est un long poème dramatique, Shelley l'a écrit avant même que les luttes en cours soient parvenues à une issue. Mais le poète a puissament perçu la valeur symbolique de ce conflit où un petit peuple, toutefois illustre par son histoire, essaie de secouer le joug que

\footnotetext{
41. Shelley Poetical Works, ed. by T. Hutchinson, a new edition, corrected by G.M. Matthews, (Oxford University Press) Oxford: 1970, p. 603-610.

42. Ibid., p. 616-620.

43. Ibid, p. 446-478.
} 
font peser sur lui non seulement l'occupant turc, mais les monarchies européennes dont l'intérêt était alors de le maintenir en servitude. Et le poète prophétise la victoire de la Grèce, la victoire de la liberté sur la tyrannie.

Il conviendrait de s'étendre beaucoup plus longuement sur ces poèmes, aussi beaux que riches de sens, et peut-être serait-il encore plus intéressant pour notre thème de relire attentivement les dix-sept lettres écrites par Shelley au sujet des mêmes circonstances ${ }^{44}$. Mais nous nous bornerons à dire brièvement ce que le poète-philosophe a vu dans ces révolutions. Essentiellement, l'éternelle bataille des opprimés contre leurs oppresseurs et contre la tyrannie : « This is the age of the war of the oppressed against the oppressors ${ }^{45}$. » En outre, il a eu un regain d'espoir en voyant les diverses classes populaires combattre pour leurs droits légitimes : «A new race has arisen throughout Europe ${ }^{46}$.» Enfin, ces mouvements révolutionnaires déclenchés par les affligés lui ont fait espérer la réalisation d'une république dont il avait commencé à rêver en lisant l'histoire des révolutions américaines et française et qu'il appelait «le beau idéal ». Il faut encore souligner un point important. Pour autant que Shelley ait été intéressé au triomphe de la révolution politique et sociale autour de lui, il n'a jamais aimé voir les hommes utiliser la violence comme moyen pour parvenir à la liberté. Toute sa vie, il l'a sévèrement condamnée, que ce soit en Irlande, en Angleterre ou ailleurs. Il a toujours essayé de faire comprendre aux hommes, soit dans ses rencontres, soit à travers ses écrits poétiques, comme The Revolt of Islam, Prometheus Unbound, The Cenci, soit dans ses essais philosophiques, soit en général dans ses lettres, que la violence n'amenait avec elle ou ne récoltait que la violence. Il a donc essayé de la combattre en propageant son idée d'association philanthropique et en essayant d'implanter cette dernière là où les mouvements révolutionnaires naissaient, par exemple, en Irlande. Comment Shelley était-il devenu un apôtre de la non-violence ? ${ }^{47}$ C'est qu'il avait tiré,

44. Voir les lettres de Shelley entre le 30 juillet 1820 et le 31 décembre 1821 .

45. Hellas, Préface, p. 448.

46. Ibia., p. 448.

47. Voir l'étude récente de M. Art Young : Sbelley and Nonviolence (Mouton Press) The Hague-Paris : 1975. Ce livre compare l'idée de non-violence chez le poète avec l'idéal et la pensée de $M$. Ghandi au début de ce siècle. 
nous dit-il dans sa préface de Revolt of Islam, une grande leçon de la Révolution française: «The French Revolution, although undertaken with the best intentions, ended ill for the people, because violence was employed. The cause which they vindicated was that of truth, but they gave it the appearance of a lie by using methods which ill-suit the purposes of liars as well as their own ${ }^{48}$. " En utilisant les mêmes moyens que ses oppresseurs, le peuple français n'a réussi qu’à moitié sa révolution.

Ce qu'il faudrait, c'est une révolution de l'esprit et du coeur qui engendre en chacun un homme nouveau, c'est-à-dire un homme éclairé par la connaissance et surtout pénétré de bienveillance envers tous les autres. Et Shelley déclare très fortement : «Ce fut une erreur de dissocier la morale de la politique, car ces deux champs ne sont que deux côtés de la même science ${ }^{49}$. $\gg$ Bref, ce n'est que dans la mesure où les hommes vivront selon les valeurs ou les idées éternelles de sagesse, d'amour et de justice qu'ils viendront à réaliser un monde meilleur, heureux et beau. Les textes que nous venons de rassembler, de résumer et d'analyser suffisent à prouver, pensons-nous, qu'il y avait déjà chez Shelley une véritable pensée socialiste. Le nom seul lui manquait, ce nom n'ayant pas encore été inventé50, mais déjà le poète en donnait par avance la définition dans une lettre sur la révolution de Naples où il dit du peuple et des paysans de Naples «qu'ils peuvent devenir les instruments d'un système futur de vie sociale destiné à supplanter l'anarchie dans laquelle la vie civile en Europe demeure pour le moment plongée ${ }^{51}$ ». Or cette idée d'un nouvel état social destiné à remplacer l'ancien dans un futur proche de nous est bien l'idée première du socialisme $e^{52}$.

Université d'Ottawa

48. Shelley Poetical Works. The Revolt of Islam, Préface, p. 33.

49. Shelley's Prose or The Trumpet of a Propbecy, "A Philosophical View of Reform », p. 253.

50. Ce mot a été inventé aux environs de 1830 par $R$. Owen et $G$. Sand.

51. The Letters of Percy Bysshe Shelley, II, no 609, p. 266-267.

52. Cet article a été présenté comme conférence à l'A.C.F.A.S. en mai 1978. 